\title{
Investigating the effect of a stress-based uniaxial anisotropy on the magnetic behaviour of $\mathrm{La}_{0.7} \mathrm{Sr}_{0.3} \mathrm{MnO}_{3}$ elements
}

\author{
K. J. O'Shea, K. Bova, D. McGrouther and D. A. MacLaren \\ SUPA, School of Physics and Astronomy, University of Glasgow, Scotland G12 8QQ
}

\begin{abstract}
We investigate the interplay between shape anisotropy and a stress-based uniaxial anisotropy on the magnetic domain structure of $\mathrm{La}_{0.7} \mathrm{Sr}_{0.3} \mathrm{MnO}_{3}$ nanoelements as a function of aspect ratio, using micromagnetic simulations. We show that a direct competition between the anisotropies gives rise to high energy multidomain flux closure configurations, whilst an alignment of the anisotropies can modify the effective element dimensions and act to stabilise a single domain configuration. Our results demonstrate the ability to control the spin state of $\mathrm{La}_{0.7} \mathrm{Sr}_{0.3} \mathrm{MnO}_{3}$ elements in addition to tailoring the domain wall width by controlling the anisotropy of the material, which is key for spintronic applications that require a high spin-polarization and stable magnetic configurations.
\end{abstract}

\section{Introduction}

$\mathrm{La}_{0.7} \mathrm{Sr}_{0.3} \mathrm{MnO}_{3}$ (LSMO) is currently the subject of increased research interest due to its attractive properties for memory applications, namely its half-metallicity and colossal magnetoresistance [1]. The study of both static and dynamic properties of magnetic domain walls in this class of materials is of significant interest for application in magnetic storage devices based on domain walls, owing to the promise of a high spin-torque efficiency for current-induced domain wall motion [2]. Its halfmetallicity also makes it an attractive material for magnetic tunnel junctions and a tunnel magnetoresistance ratio of $1800 \%$ [3] has previously been reported. However, it has been shown that the magnetic domain structure of LSMO depends on a number of factors including substrate-induced strain [4] and doping level [5]. It is therefore critical to fully understand the dependence of the magnetic behaviour on these different parameters. It has previously been shown that when grown on STO(110) substrates, a stress-based uniaxial anisotropy is induced in LSMO films whilst a biaxial anisotropy is observed for LSMO grown on (001)oriented STO substrates. It has been shown that the addition of a biaxial anisotropy to interacting Co nanoislands enhances their hard axis stability [6] and consequently may be exploited in magnetic logic circuits. Here, we focus on the magnetic behaviour of LSMO films on (110)-oriented STO substrates and use micromagnetic simulations to show that by combining the shape anisotropy with a uniaxial anisotropy, it is possible to tailor the magnetic domain structure and ultimately the domain wall width.

\section{Micromagnetic simulation}

The Object Oriented MicroMagnetic Framework (OOMMF) [7] was used to calculate the magnetic domain structure of LSMO nanoelements. The simulations used a $5 \mathrm{~nm}$ mesh, (i.e. comparable to the magnetostatic exchange length [8]) and employed the following magnetic parameters for a (110)-oriented STO substrate [9]: saturation magnetization, $\mathrm{Ms}=5.6 \times 10^{5} \mathrm{~A} / \mathrm{m}$; exchange stiffness $\mathrm{A}=1.86 \times 10^{-12} \mathrm{~J} / \mathrm{m}$; and uniaxial strain anisotropy constant, $\mathrm{K}_{\mathrm{U}}=1.9 \times 10^{4} \mathrm{~J} / \mathrm{m}^{3}$. The Gilbert damping constant was set to $\alpha=0.5$ to allow rapid convergence in calculations. Technologies based on nanomagnets typically have critical dimensions below $200 \mathrm{~nm}$ [10], so a range of rectangular thin film elements were investigated, where the breadth was kept constant at $160 \mathrm{~nm}$, and the length varied in integer multiples of the width to explore the effect of aspect ratio. The uniaxial anisotropy, $\mathrm{K}_{\mathrm{U}}$, is directed along the [001] axis, with the hard axis being along [1 $1 \overline{1} 0]$, as illustrated in figure 1 . Hereafter, $\theta_{\mathrm{LA}}$ will denote the relative orientation of the element long axis with respect to the [001] easy direction. In the following sections, we will illustrate the lowest energy static configurations supported by the elements, when $\theta_{\mathrm{LA}}=0^{\circ}$ and $\theta_{\mathrm{LA}}=90^{\circ}$. The magnetic behaviour will then be discussed and is shown to be highly dependent on $\theta_{\text {LA }}$. Finally, we will illustrate the effect of $\mathrm{K}_{\mathrm{U}}$ on the resulting transverse and vortex domain wall widths in LSMO nanowires. 


\section{Ground states}

Figures 1a and c summarise the lowest energy configurations for an increasing aspect ratio of LSMO elements on (110)-oriented substrates, for $\theta_{\mathrm{LA}}=0^{\circ}$ and $\theta_{\mathrm{LA}}=90^{\circ}$, respectively. The elements were relaxed from a variety of starting conditions to assess the range of different configurations that may be supported. In figure 1a, the introduction of the uniaxial anisotropy in the square element distorts the 4-fold symmetry and results in an asymmetric Landau state, where the two horizontal domains (red and blue regions) are slightly enlarged at the expense of the vertically aligned domains (white regions). The single vortex configuration is the lowest in energy until an aspect ratio of $3: 1$ is reached, where a near single domain state is favoured. By forming a single domain state rather than a vortex structure, the system reduces its total energy by $7 \%$ (not shown here). As indicated at $\mathbf{A}$ in figure 1b, the exchange and anisotropy energies significantly decrease due to the absence of multiple domain walls, and the magnetostatic energy increases considerably as a result of the increased stray field being generated at the element ends (at $\mathbf{B}$ in figure 1b). On increasing the element aspect ratio further, the further increase in total energy is significantly smaller; the total energy of the 10:1 element is only $4.7 \%$ higher than the 3:1 element, since it is dominated by the magnetostatic energy contribution, which hardly changes.

Results for $\theta_{\mathrm{LA}}=90^{\circ}$ are presented in figure $1 \mathrm{c}$, where the square in figure $1 \mathrm{c}$ is identical to that in figure $1 \mathrm{a}$, as would be intuitively expected. As the length increases in integer multiples of the width, rather than the single vortex state of figure 1a, here the number of vortices also increases, up to at least 10 for a 10:1 aspect ratio element. Although a number of different multidomain configurations may be supported, including a single vortex and triple vortex state in the 2:1 element for example, the minimum energy configuration consists of a single vortex per integer multiple of width, and the
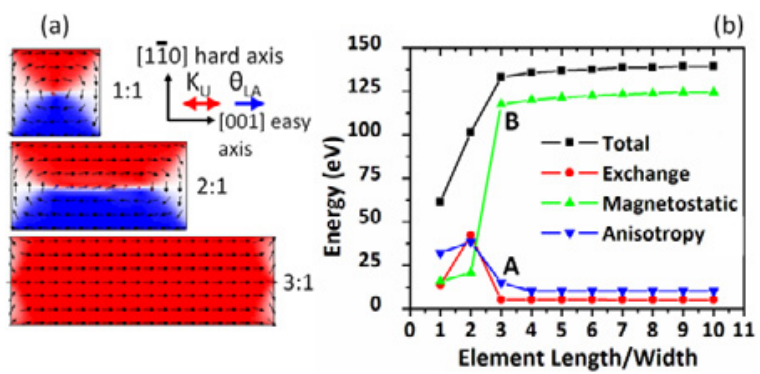

(c)
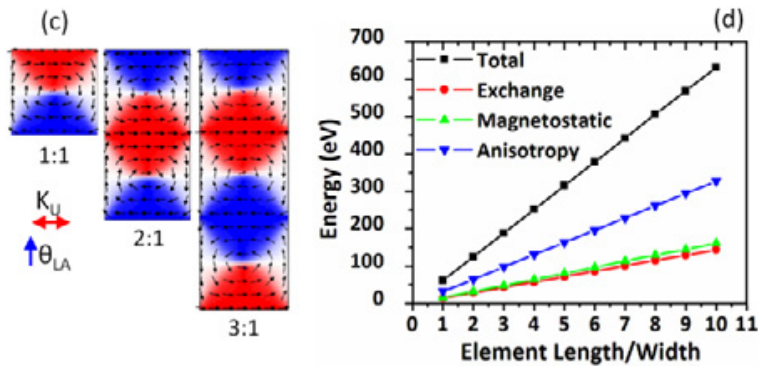

Fig. 1. Effect of increasing the aspect ratio with (a) $\theta_{\mathrm{LA}}=0^{\circ}$ and (c) $\theta_{\mathrm{LA}}=90^{\circ}$. The total energy is also presented in (b), (d) as a function of aspect ratio. total energy scales linearly with element length (figure 1d). The total energy for $\theta_{\mathrm{LA}}=90^{\circ}$, where $\mathrm{K}_{\mathrm{U}}$ opposes the shape anisotropy, is considerably higher than that for $\theta_{\mathrm{LA}}=90^{\circ}$, where the shape anisotropy reinforces $\mathrm{K}_{\mathrm{U}}$.

\section{Magnetic reversal behaviour}

\section{1. $K_{U}$ parallel to shape anisotropy}

It is critical to understand the effect of the uniaxial anisotropy on the magnetic reversal behaviour of thin film elements. In the following sections, we present the magnetisation reversal for both $\theta_{\mathrm{LA}}=0^{\circ}$ and $\theta_{\mathrm{LA}}=90^{\circ}$ for a magnetic field along the [001] and [11̄0] axes. For $\theta_{\mathrm{LA}}=0^{\circ}$, the behaviour of a rectangular element of aspect ratio $7: 1$ is comparable to a typical soft ferromagnetic element such as permalloy [11]. As illustrated by the black curve in figure $2 \mathrm{a}$, as the magnetic field along the easy [001] direction is reduced to $0 \mathrm{mT}$, small reverse domains form at either end of the element, shown immediately prior to reversal at $\mathbf{C}$ in figure $2 \mathrm{~b}$. Expansion of the reverse domains prior to reversal is energetically unfavourable in LSMO as a result of the presence of $\mathrm{K}_{\mathrm{U}}$ and as such, magnetization reversal is abrupt at $-40 \mathrm{mT}$ (at $\mathbf{D}$ in figure $2 \mathrm{~b}$ ).

The deviation from a typical soft ferromagnet is more marked for a magnetic field applied perpendicular to the

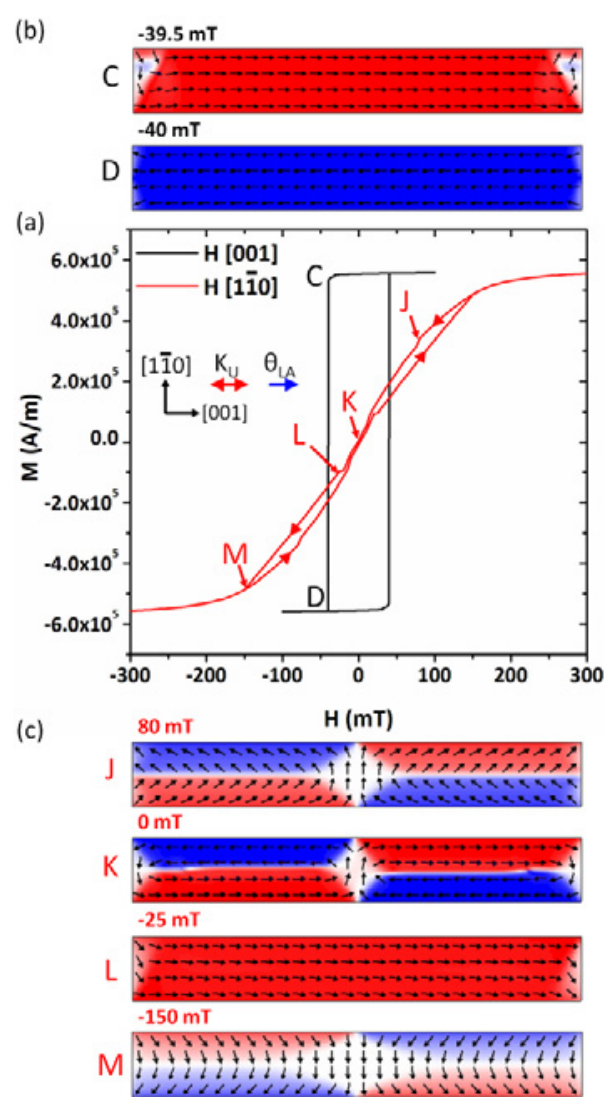

Fig. 2. (a) Hysteresis loops of 7:1 aspect ratio LSMO nanoelements where $\theta_{\mathrm{LA}}=0^{\circ}$. A magnetic field applied along the [001] direction is given by the black curve and a magnetic field applied along [110] is given by the red curve. The magnetic configuration at each stage is given in (b) and (c) for a magnetic field along [001] and [1 10$]$, respectively. 
shape and uniaxial anisotropies, and is illustrated by the red curve in figure 2a. A 5-domain configuration is formed on reducing the magnetic field from saturation (at $\mathbf{J}$ in figure 2c), however the presence of the shape anisotropy forces the spins to lie parallel to the wire axis at remanence (indicated by the saturated red and blue regions at $\mathbf{K}$ in figure $2 \mathrm{c}$ ). There is a considerable exchange energy cost to the formation of the long domain walls in the configuration at $\mathbf{K}$. Thus the system energy is minimised by the formation of the single domain state at $\mathbf{L}$ in figure $2 \mathrm{c}$, which reduces the exchange energy by $98 \%$ and the anisotropy energy by $68 \%$; this is almost balanced by a $66 \%$ increase in magnetostatic energy. At $-150 \mathrm{mT}$, the field is sufficient to overcome both the uniaxial and shape anisotropies and a near single domain structure is formed (at $\mathbf{M}$ in figure 2c).

\section{2. $K_{U}$ perpendicular to shape anisotropy}

The magnetic reversal behaviour is somewhat different for $\theta_{\mathrm{LA}}=90^{\circ}$, as illustrated in figure $3 \mathrm{a}$. Here the applied magnetic field is parallel to the [001] easy axis. On reducing the field from saturation, a combination between a flower state and a diamond state [12] is formed at a field of $10 \mathrm{mT}$ (at $\mathbf{N}$ in figure $3 \mathrm{~b}$ ). The large step immediately following $\mathbf{N}$ in figure $3 b$ is a result of the element forming a 4-vortex state from the flower state at $\mathbf{N}$. At remanence (at $\mathbf{O}$ in figure $3 b$ ) the lowest energy configuration, a 7-vortex state determined in figure $1 \mathrm{c}$, is not accessible and instead a 4-vortex state is formed, which is $11 \%$ higher in energy. This is a result of the magnetic field being parallel to $\mathrm{K}_{\mathrm{U}}$, which promotes the

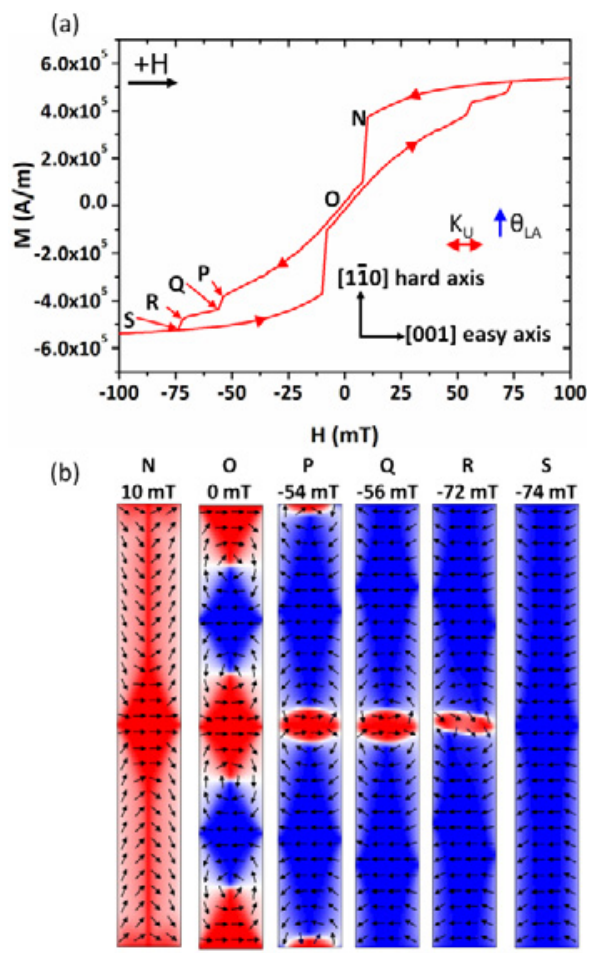

Fig. 3. (a) Hysteresis loop of the 7:1 element with $\theta_{\mathrm{LA}}=90^{\circ}$ and a magnetic field applied along the [001] easy direction. The magnetic configuration at each critical stage is given in (b). A higher energy 4-vortex state is produced at remanence as a result of the field direction. alignment of a higher proportion of spins along this axis at remanence. On increasing the reverse field, the domains parallel to the field direction (blue domains) expand at the expense of those anti-parallel (red domains at $\mathbf{P}$ in figure $3 b$ ) until two of the four vortices are expelled at a field of $-56 \mathrm{mT}$ (at $\mathbf{Q}$ in figure $3 \mathrm{~b}$ ). By a field of $-72 \mathrm{mT}$ (at $\mathbf{R}$ in figure $3 \mathrm{~b}$ ), the central domain aligned anti-parallel to the applied field begins to reverse and at a field of -78mT a near-single domain state is reached (at $\mathbf{S}$ in figure $3 b)$.

The effect of a magnetic field applied along the [110] hard direction is presented in figure 4a. On reducing the field from saturation, a vortex is nucleated at either end to create an S-vortex state at a field of $30 \mathrm{mT}$ ( $\mathbf{T}$ in figure $4 \mathrm{~b})$. Two additional vortices are nucleated immediately adjacent to the original vortices at $15 \mathrm{mT}$ (at $\mathbf{U}$ in figure $4 b$ ). Additionally, the central region of the element is no longer uniform and a concertina-like pattern is formed as a result of the competing anisotropies. At a field of $10 \mathrm{mT}$ (at $\mathbf{V}$ in figure $4 \mathrm{~b}$ ), four additional vortices have been nucleated, thus forming an 8 -vortex configuration at remanence (at $\mathbf{W}$ in figure $4 b$ ). From the data presented in figure 1d, a 7-vortex state is the lowest energy configuration for a 7:1 aspect ratio element; however this is not accessible via this magnetic field sequence. Since pairs of vortices are nucleated from the element ends as the field is reduced, an odd number of vortices is prohibited in the remanent state. Interestingly, the 8 -domain state is only $3 \%$ higher in energy than the 7-domain state, thus it is easily reached. On increasing the field in the opposite direction, the vortices are forced towards the element edges as the domains parallel with the field expand (at $\mathbf{X}$ in figure $4 b$ ).
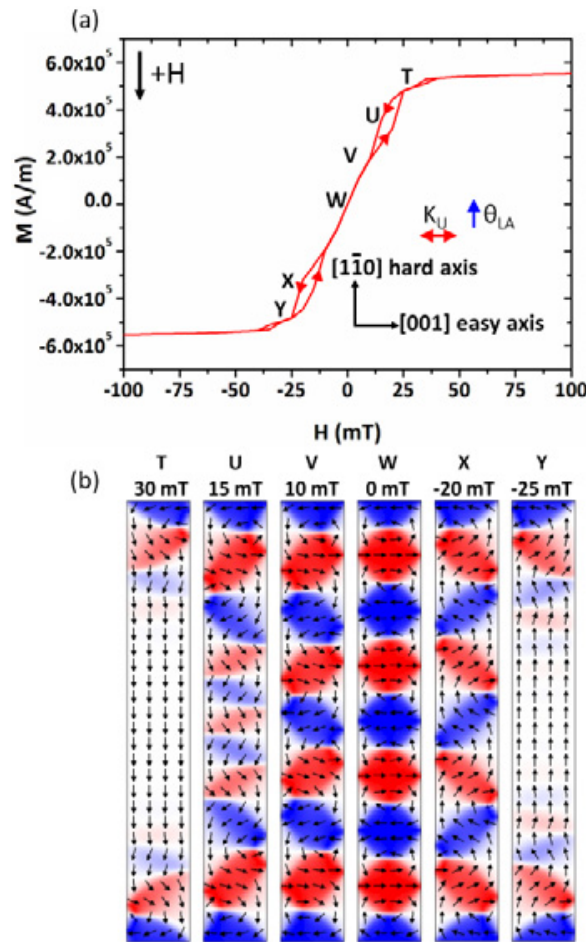

Fig. 4. (a) Hysteresis loop of the 7:1 element with $\theta_{\mathrm{LA}}=90^{\circ}$ and a magnetic field applied along the [1 $\overline{1} 0]$ hard direction. (b) The magnetisation is displayed at various stages. An odd number of vortices is prohibited at remanence, thus a slightly higher energy 8 -vortex state is produced. 
At a field of $-25 \mathrm{mT}$, six of the eight vortices are expelled from the element, creating an S-vortex state once again (at $\mathbf{Y}$ in figure $4 b$ ).

\section{Domain Wall Width}

It has recently been shown that the addition of a uniaxial anisotropy contribution in permalloy nanowires can either increase or decrease the domain wall width [13]. Walker breakdown effects can also be suppressed and a $30 \%$ increase in domain wall velocity was reported in a similar system when the in-plane anisotropy was oriented perpendicular to the wire long axis [14]. We investigate the effect of $\mathrm{K}_{\mathrm{U}}$ on the magnetic domain wall width for $1120 \times 160 \times 10 \mathrm{~nm}$ nanowires, which is summarized in figure 5. To remove the effect of any complex end domain structures on the resulting domain wall structure, the stray field from $2 \mu \mathrm{m}$ long tracks either side of the wire was included in the simulation as a fixed Zeeman field to act as a continuation of the wire. In effect, this removes the demagnetizing field from the ends of the wire. Given that the magnetization profile differs substantially with the addition of a uniaxial anisotropy, and differs significantly from a transverse to vortex structure, we define the domain wall width to be the region enclosed by $\mathrm{M}_{\mathrm{x}} / \mathrm{M}_{\mathrm{s}}= \pm 0.95$, thus allowing a direct comparison of domain wall widths, which is independent of wall structure [13]. Without a uniaxial anisotropy, the width of a transverse domain wall, $\Delta \mathrm{TW}$, was measured to be $125 \mathrm{~nm}$, and the width of a vortex domain wall, $\triangle \mathrm{VW}$, was measured to be $258 \mathrm{~nm}$ in a LSMO nanowire. However, with a uniaxial anisotropy term, the width of the transverse domain wall, $\Delta \mathrm{TW}$, is reduced by $45 \%$ to $69 \mathrm{~nm}$ and the vortex domain wall width, $\Delta \mathrm{VW}$, is reduced by $30 \%$ to $182 \mathrm{~nm}$, respectively. Essentially, the uniaxial anisotropy acts with the shape anisotropy to alter the effective wire dimensions [13].

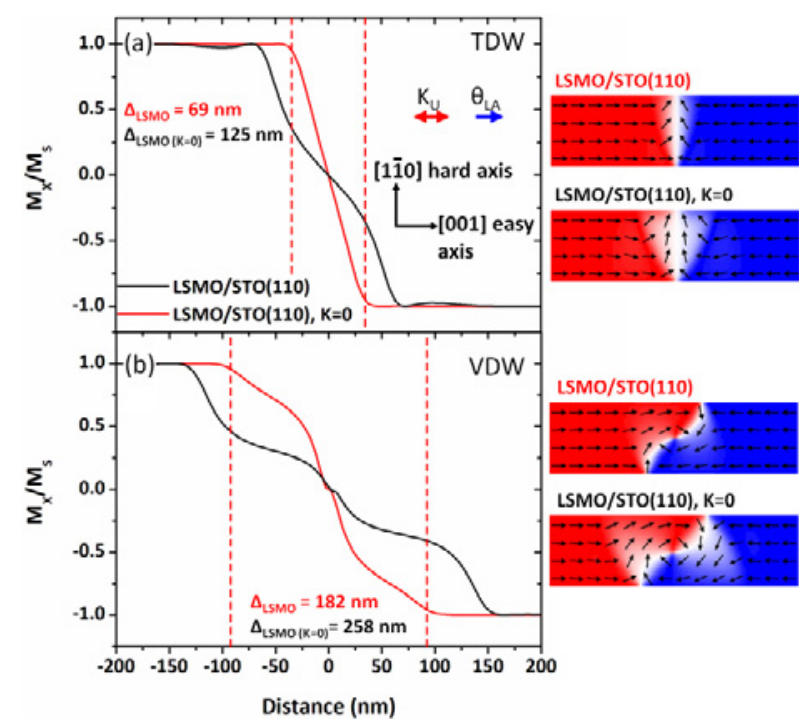

Fig. 5. The normalized $x$-component of magnetization through the centre of (a) a transverse and (b) a vortex domain wall in a LSMO/STO(110) nanowire (red curve). Red dashed lines indicate the extent of the domain wall. A comparison with LSMO where $\mathrm{K}=0$ (black curve) is also included.

\section{Summary and conclusions}

In summary, we have shown that for LSMO elements with orthogonal shape and uniaxial anisotropies, it is possible to control the number of vortices by simply varying the aspect ratio, and ultimately the shape anisotropy. Further control of the magnetic vortex density is achieved by the application of a magnetic field. With a favourable alignment of the shape and uniaxial anisotropies, the behaviour is comparable to that of a typical soft ferromagnetic element such as permalloy, with the uniaxial anisotropy largely acting to alter the coercivity. Moreover, a uniaxial anisotropy was shown to reduce both the transverse and vortex domain wall width, highlighting the possibility to tailor domain walls by controlling the material anisotropy during fabrication. Ultimately, LSMO can provide the advantages of a soft ferromagnetic material with the capacity for stable multivortex configurations, along with the capability to act as a source of fully spin-polarized current which makes it a promising candidate for both the study of fundamental domain wall phenomena in addition to device applications.

The authors acknowledge financial support from the European Research Council under the 7th Framework Program (FP7), Grant No. NMP3-LA-2010-246102 IFOX.

\section{References}

1. A.-M. Haghiri-Gosnet, J.-P. Renard, J. Phys. D: Appl. Phys. 36, R127 (2003)

2. A. Thiaville, Y. Nakatani, J. Miltat, Y. Suzuki, Europhys. Lett. 69, 990 (2005)

3. M. Bowen, M. Bibes, A. Barthélémy, J.-P . Contour, A. Anane, Y. Lemaître, A. Fert, Appl. Phys. Lett. 82, $233(2003)$

4. Y. Suzuki, H. Y. Hwang, S.-W. Cheong, R. B. Van Dover, Appl. Phys. Lett. 71, 140 (1997)

5. T. Koyama, Y. Togawa, K. Takenaka, S. Mori, J. Appl. Phys. 111, 07B104 (2012)

6. D. B. Carlton, N. C. Emley, E. Tuchfeld, J. Bokor, Nano Lett. 8, 4173 (2008)

7. http://www.nist.gov

8. A. P. Guimarães, Principles of Nanomagnetism (Springer, 2009)

9. L. M. Berndt, V. Balbarin, Y. Suzuki, Appl. Phys. Lett. 77, 2903 (2000)

10. B. D. Terris, T. Thomson, G. Hu, Microsyst. Technol. 13, 189 (2006)

11. A. Hubert, R. Schäfer, Magnetic Domains: The Analysis of Magnetic Microstructure, (Springer, 2009)

12. W. Rave, A. Hubert, IEEE Trans. Magn. 36, 3886 (2000)

13. M. T. Bryan, S. Bance, J. Dean, T. Schrefl, D. A. Allwood, J. Phys.: Condens. Matter 24, 024205 (2012)

14. J. S. Dean, M. T. Bryan, D. A. Allwood, S. Bance, M. A. Bashir, G. Hrkac, A. Goncharov, T. Schrefl, IEEE Trans. Magn. 454067 (2009) 